\title{
Sum rule expressions for bounds on van der Waals coefficients.
}

\author{
R. Luyckx, ${ }^{\text {a) }} \mathrm{Ph}$. Coulon, and H. N. W. Lekkerkerker \\ Faculteit van de Wetenschappen, Vrije Universiteit Brussel, 1050 Brussel, Belgium \\ (Received 26 January 1979)

\begin{abstract}
We present a simple matrix solution for the moment equations that occur in recently discovered bounds on van der Waals coefficients. Using this matrix solution it is possible to express these new bounds directly in terms of the oscillator strength sum rules of the interacting systems.
\end{abstract}

\section{INTRODUCTION}

About ten years ago, Langhoff and Karplus ${ }^{1-3}$ and Gordon ${ }^{4}$ reported, for the first time, methods for obtaining upper and lower bounds for van der Waals coefficients. These bounds were constructed by using, in the Casimir-Polder integral formula,${ }^{5}$ approximate dy namic polarizabilities that are upper or lower bounds to the exact dynamic polarizabilities at imaginary frequencies. These bounded dynamic polarizabilities were obtained by recognizing that the series expansion of $\alpha(i \xi)$ in positive powers of $\xi$ is a series of Stieltjes (the expansion coefficients being the negative even moments of the oscillator strength distribution) and using results from the theory of Pade approximants or employing elements of Gaussian integration theory. It has been shown that these two methods are equivalent.$^{6}$

The inequalities mentioned above involve only even sum rules. To some extent, this may be considered to be an advantage since, for the dipole case, these sum rules can be obtained from optical refractivity data. However, this is not the case for the higher order multipole polarizabilities, where one has to resort to theoretical calculations. These calculations, which become increasingly feasible at the present time, yield both even and odd sum rules. Therefore, the question arises whether it is possible to construct bounds on the van der Waals coefficients using both even and odd sum rules. An elegant general method for doing this has been worked out by Alexander. ${ }^{8}$ However, his procedure requires the knowledge of the lowest excitation frequency and, more seriously, the bounds provided by it are not optimal. Further, Langhoff ${ }^{9}$ has obtained upper and lower bounds on van der Waals coefficients using the sum rules $S(2), S(1), S(0), S(-1), S(-2)$, and the lowest excitation frequency.

Recently, we ${ }^{10}$ have been able to obtain bounds on van der Waals coefficients, using even and odd sum rules, which are both general and optimal. Here we show that the moment equations that occur in the formulation of these new bounds can be solved by a simple matrix method. Further, this matrix solution allows one to express the bounds on the van der Waals coefficients directly in terms of sum rules.

\footnotetext{
a)Aspirant Nationaal Fonds voor Wetenschappelijk Onderzoek. (N. F. W. O.) Belgium.
}

\section{BOUNDS ON VAN DER WAALS COEFFICIENTS}

In this section, we briefly state the principal results obtained in Ref. 10, which at the same time serve to establish the notation. The leading term of the dispersion interaction between two spherically symmetric atoms $\mathrm{A}$ and $\mathrm{B}$ at distance $R$ can be written as

$$
U_{\mathrm{AB}}=-C_{\mathrm{AB}} / R^{6} \text {, }
$$

with

$$
C_{\mathrm{AB}}=\frac{3}{\pi} \int_{0}^{\infty} \alpha_{\mathrm{A}}(i \xi) \alpha_{\mathrm{B}}(i \xi) d \xi .
$$

Here, $\alpha(i \xi)$ is the dynamic dipole polarizability

$$
\alpha(i \xi)=\int_{0}^{\infty} \frac{d \psi(u)}{u^{2}+\xi^{2}},
$$

where $d \psi(u)$ is the oscillator strength density between $u$ and $u+d u$. Substituting the dynamic polarizability given by $\mathrm{Eq}$. (2) in (1), one obtains

$$
C_{\mathrm{AB}}=\frac{3}{2} \int_{0}^{\infty} d \psi_{\mathrm{A}}(u) \int_{0}^{\infty} d \psi_{\mathrm{B}}(v) \frac{1}{u v(u+v)} .
$$

We now construct an approximate oscillator strength distribution

$$
d \bar{\psi}(u)=\sum_{i=1}^{n} \bar{f}_{i} \delta\left(u-\bar{u}_{i}\right) d u,
$$

where the weights $\bar{f}_{i}$ and positions $\bar{u}_{i}$ are determined by the condition that $d \psi(u)$ reproduces $2 n$ oscillator strength sum rules

$$
\begin{aligned}
& \int_{0}^{\infty} d \bar{\psi}(u) u^{k}=\int_{0}^{\infty} d \psi(u) u^{k}=S(k), \\
& k=-q,-q-1, \ldots,-q-2 n+1,
\end{aligned}
$$

where ${ }^{11} q=-2,-1,0,1,2,3, \ldots$, and $n=1,2,3, \ldots$.

At this point, we would like to emphasize that the approximate polarizabilities $\bar{\alpha}(i \xi)$ which one obtains by substituting $d \bar{\psi}(u)$ in Eq. (2) are neither upper nor lower bounds to the exact polarizability at imaginary frequencies. Still, it is possible to show ${ }^{10}$ that the approximate van der Waals coefficients

$$
\bar{C}_{\mathrm{AB}}=\frac{3}{2} \int_{0}^{\infty} d \bar{\psi}_{\mathrm{A}}(u) \int_{0}^{\infty} d \bar{\psi}_{\mathrm{B}}(v) \frac{1}{u v(u+v)}
$$

are upper or lower bounds depending on which sum rules are used for the construction of the approximate oscillator strength densities. Indeed, the approximate oscillator strength densities can be divided into two groups, 
where group I comprises the cases $q=-2,0,2,3,4, \ldots$, with $n=1,2,3, \ldots$, and $q=-1, n=1$ and group II the remaining cases, i.e., $q=-1, n=2,3,4, \ldots$, and $q=1$, $n=1,2,3, \ldots$, such that the van der Waals coefficients calculated with approximate oscillator strength densities which belong to group I are lower bounds, whereas those calculated with group II are upper bounds.

\section{MATRIX SOLUTION FOR THE MOMENT EQUATIONS}

In actual calculations of the bounds, one has to obtain the strength factors $\bar{f}_{i}$ and effective excitation energies $\bar{u}_{i}$ from the equations one obtains by substituting Eq. (4) in (5):

$$
\sum_{i=1}^{n} \bar{f}_{i} \bar{u}_{i}^{-k}=S(-k), \quad k=q, q+1, \ldots, q+2 n-1 .
$$

A matrix solution for this kind of equations has been given by Gordon. ${ }^{12}$ His method proceeds in two main stages. First, the moments are transformed into a set of coefficients that appear in the continued fraction expansion. Subsequently, these coefficients are used to construct a matrix from whose eigenvalues and eigenvectors the strength factors and effective excitation energies can be expressed. Here we present an alternative matrix method that proceeds directly from the sum rules to the strength factors and effective excitation energies.

Consider the $n \times n$ matrices $\mathbf{M}$ and $\mathbf{N}$ defined as

$$
\begin{aligned}
& M_{i j}=S(-q-i-j+2) \\
& N_{i j}=S(-q-i-j+1), \quad i, j=1,2,3, \ldots, n .
\end{aligned}
$$

We now introduce the matrix

$$
A=\mathbf{N}^{-1} \mathbf{M} \text {, }
$$

which, since $\mathbf{N}$ and $\mathbf{M}$ are real symmetric positive definite matrices, has positive real eigenvalues and $n$ linearly independent eigenvectors. In the Appendix, we will show that

$$
\left[\mathbf{N A}^{-l}\right]_{11}=S(-q-l-1), \quad l=-1,0,1,2, \ldots, 2 n-2 .
$$

The strength factors $\bar{f}_{i}$ and effective excitation energies $\bar{u}_{i}$ can be expressed in terms of the eigenvalues $\lambda_{i}$ and corresponding residue matrices $\mathbf{R}^{(i)}$ of the matrix A as follows:

$$
\begin{aligned}
& \bar{u}_{i}=\lambda_{i}, \\
& \bar{f}_{i}=\lambda_{i}^{(1+q)}\left[\mathbf{N R}^{(i)}\right]_{11} .
\end{aligned}
$$

The residue matrices $\mathbf{R}^{(i)}$ are given by

$$
\mathbf{R}^{(i)}=\mathrm{V}_{i} \mathrm{~W}_{i} .
$$

Here, $V_{i}$ is the right (column) eigenvector and $W_{i}$ is the left (row) eigenvector of $\mathbf{A}$ corresponding to the eigenvalue $\lambda_{i}$, which are normalized such that

$$
\mathrm{W}_{i} \cdot \mathrm{V}_{i}=1, i=1,2, \ldots, n .
$$

Using Eq. (10) and the well-known property of residue matrices

$$
\sum_{i=1}^{n} \lambda_{i}^{l} \mathbf{R}^{(i)}=\mathbf{A}^{l},
$$

where $l$ is a positive or negative integer or zero, one easily verifies that $\bar{f}_{i}$ and $\bar{u}_{i}$ as given by Eq. (11) indeed satisfy the moment equations (7):

$$
\begin{aligned}
\sum_{i=1}^{n} \bar{f}_{i}\left(\bar{u}_{i}\right)^{-k} & =\sum_{i=1}^{n} \lambda_{i}^{1+\sigma^{-k}}\left[\mathbf{N} \mathbf{R}^{(i)}\right]_{11} \\
& =\left[\mathbf{N A}^{-\left(k-\sigma^{-1)}\right.}\right]_{11} \\
& =S(-k), \quad k=q, q+1, \ldots, q+2 n-1 .
\end{aligned}
$$

Given the oscillator strength sum rules, the matrix method presented here allows one to obtain the strength factors $\bar{f}_{i}$ and excitation energies $\bar{u}_{i}$ directly by applying standard matrix inversion and diagonalization procedures.

\section{SUM RULE EXPRESSIONS FOR BOUNDS ON VAN DER WAALS COEFFICIENTS}

Here we wish to show that the matrix solution presented in the previous section allows one to express the bounds on the van der Waals coefficients directly in terms of the sum rules without the intermediate calculation of strength factors and effective excitation energies. Substituting the approximate dynamic polarizabilities characterized by the oscillator strength distribution (4) in Eq. (6), one obtains

$$
\bar{C}_{\mathrm{AB}}=\frac{3}{2} \sum_{i=1}^{n} \sum_{j=1}^{m} \frac{\bar{f}_{i} \bar{g}_{j}}{\bar{u}_{i} \bar{v}_{j}\left(\bar{u}_{i}+\bar{v}_{j}\right)} .
$$

Here, $\bar{f}_{i}$ and $\bar{u}_{i}$ are the strength factors and effective excitation energies of atom $\mathrm{A}$ and $\bar{g}_{j}$ and $\bar{v}_{j}$ those of atom B. Using the expressions (11) for the quantities, the van der Waals coefficient can be written as

$$
C_{\mathrm{AB}}=\frac{3}{2} \sum_{i=1}^{n} \sum_{j=1}^{m} \frac{\lambda_{i}^{q} \mu_{j}^{p}\left[\mathbf{N}_{\mathrm{A}} \mathbf{R}_{\mathrm{A}}^{(i)}\right]_{11}\left[\mathbf{N}_{\mathrm{B}} \mathbf{R}_{\mathrm{B}}^{(i)}\right]_{11}}{\left(\lambda_{i}+\mu_{j}\right)} .
$$

Realizing that $\lambda_{i}^{q} \mu_{j}^{p} /\left(\lambda_{i}+\mu_{j}\right)$, with $i=1,2, \ldots, n$ and $j=1$, $2, \ldots, m$, are the eigenvalues of the $n m \times n m$ matrix

$$
\left[\mathbf{N}_{\mathrm{A}}^{-1} \mathbf{M}_{\mathrm{A}} \otimes \mathbf{I}_{m}+\mathbf{I}_{n} \otimes \mathbf{N}_{\mathrm{B}}^{-1} \mathbf{M}_{\mathrm{B}}\right]^{-1}\left[\left(\mathbf{N}_{\mathrm{A}}^{-1} \mathbf{M}_{\mathrm{A}}\right)^{q} \otimes\left(\mathbf{N}_{\mathrm{B}}^{-1} \mathbf{M}_{\mathrm{B}}\right)^{\phi}\right]
$$

and that the corresponding residue matrices are given by

$$
\mathbf{R}_{\mathrm{A}}^{(i)} \otimes \mathbf{R}_{\mathrm{B}}^{(j)},
$$

the van der Waals coefficient can be written as

$$
\begin{aligned}
\bar{C}_{\mathrm{AB}}= & \frac{3}{2}\left\{\left[\left(\mathbf{N}_{\mathrm{A}}^{-1} \mathbf{M}_{\mathrm{A}}\right)^{1-q} \mathbf{N}_{\mathrm{A}}^{-1} \otimes\left(\mathbf{N}_{\mathrm{B}}^{-1} \mathbf{M}_{\mathrm{B}}\right)^{-\phi} \mathbf{N}_{\mathrm{B}}^{-1}\right.\right. \\
& \left.\left.+\left(\mathbf{N}_{\mathrm{A}}^{-1} \mathbf{M}_{\mathrm{A}}\right)^{-q} \mathbf{N}_{\mathrm{A}}^{-1} \otimes\left(\mathbf{N}_{\mathrm{B}}^{-1} \mathbf{M}_{\mathrm{B}}\right)^{1-\rho}\right]^{-1}\right\}_{11} .
\end{aligned}
$$

The advantage of the above formula is that it expresses the van der Waals coefficients directly in terms of the sum rules of the interacting systems. If $(q, n)$ and $(p, m)$ belong to group I, the approximate van der Waals coefficient $\bar{C}_{\mathrm{AB}}$ given by Eq. (15) will be a lower bound, whereas if $(q, n)$ and $(p, m)$ belong to group $I$, the $\bar{C}_{\mathrm{AB}}$ will be an upper bound.

Special important cases are the lower bounds $q=p=0$, $n, m=1,2, \ldots$, which make use of the sum rules $S_{\mathrm{A}}(0)$, $S_{\mathrm{A}}(-1), \ldots, S_{\mathrm{A}}(-2 n+1)$ for atom $\mathrm{A}$ and $S_{\mathrm{B}}(0), S_{\mathrm{B}}(-1), \ldots$, $S_{\mathrm{B}}(-2 m+1)$ for atom B. The general expression (15) then reduces to 


$$
C_{\mathrm{AB}}=\frac{3}{2}\left[\left(\mathbf{N}_{\mathrm{A}}^{-1} \mathbf{M}_{\mathrm{A}} \mathbf{N}_{\mathrm{A}}^{-1} \otimes \mathbf{N}_{\mathrm{B}}^{-1}+\mathbf{N}_{\mathrm{A}}^{-1} \otimes \mathbf{N}_{\mathrm{B}}^{-1} \mathbf{M}_{\mathrm{B}} \mathbf{N}_{\mathrm{B}}^{-1}\right)^{-1}\right]_{11} .
$$

The matrices $\mathbf{M}$ and $\mathbf{N}$ are defined in terms of the sum rules according to Eq. (8). Using the sum rules $S_{\mathrm{A}}(-1)$, $S_{\mathrm{A}}(-2), \ldots, S_{\mathrm{A}}(-2 n)$ and $S_{\mathrm{B}}(-1), S_{\mathrm{B}}(-2), \ldots, S_{\mathrm{B}}(-2 m)$, one obtains the upper bound $q=p=1, n, m=1,2, \ldots$.

The general expression (15) then reduces to

$$
C_{\mathrm{AB}}=\frac{3}{2}\left[\left(\mathbf{N}_{\mathrm{A}}^{-1} \otimes \mathbf{M}_{\mathrm{B}}^{-1}+\mathbf{M}_{\mathrm{A}}^{-1} \otimes \mathbf{N}_{\mathrm{B}}^{-1}\right)^{-1}\right]_{11},
$$

where the matrices $\mathbf{M}$ and $\mathbf{N}$ are again defined according to $\mathrm{Eq}$. (8).

\section{ACKNOWLEDGMENT}

One of us (R.L.) would like to thank the "Nationaal Fonds voor Wetenschappelijk Onderzoek (Belgium)" for financial support.

\section{APPENDIX}

For $l=-1$ and $l=0$, the result expressed by Eq. (15) can be verified by direct substitution. For $l=1,2, \ldots$, $2 n-2$, we write

$$
\left[\mathbf{N A}^{-l}\right]_{11}=\left[\left(\mathbf{N M}^{-1}\right)^{r} \mathbf{N}\left(\mathbf{M}^{-1} \mathbf{N}\right)^{s}\right]_{11},
$$

with $r+s=l$ and $r, s=0,1, \ldots, n-1$. One easily sees that, for $r, s=0,1, \ldots, n-1$,

$\left(\mathbf{N M}^{-1}\right)_{1 j}^{r}=\delta_{j, r+1}$,

$$
\left(\mathbf{M}^{-1} \mathbf{N}\right)_{j 1}^{s}=\delta_{j, s+1} .
$$

Using Eqs. (A2) and (A3) in the righthand side of Eq. (A1), one obtains

$$
\left[\mathrm{NA}^{-l}\right]_{11}=N_{r+1, s+1}=S(-q-r-s-1)=S(-q-l-1) .
$$

${ }^{1}$ P. W. Langhoff and M. Karplus, Phys. Rev. Lett. 19, 1461 (1967).

${ }^{2}$ P. W. Langhoff and M. Karplus, J. Chem. Phys. 53, 233 (1970).

${ }^{3}$ P. W. Langhoff and M. Karplus, "Applications of Pade Approximants to Dispersion Force and Optical Polarizability Computations," in The Pade Approximant in Theoretical Physics, edited by G. A. Baker, Jr. and J. L. Gammel (Academic, New York, 1970), pp. 41-97.

${ }^{4}$ R. G. Gordon, J. Chem. Phys. 48, 3929 (1968).

${ }^{5}$ H. B. G. Casimir and D. Polder, Phys. Rev. 73, 455 (1948).

${ }^{6}$ P. W. Langhoff, R. G. Gordon, and M. Karplus, J. Chem. Phys. 55, 2126 (1971).

${ }^{7}$ P. W. Langhoff and M. Karplus, J. Opt. Soc. Am. 59, 863 (1969).

${ }^{8}$ M. H. Alexander, Phys. Rev. A 1, 1397 (1970).

${ }^{9}$ P. W. Langhoff, Chem. Phys. Lett. 12, 217 (1971).

${ }^{10}$ R. Luyckx, F. Delbaen, Ph. Coulon, and H. N. W. Lekkerkerker, Phys. Rev. A 19, 324 (1979).

${ }^{11}$ Oscillator strength sum rules $S(k)$ with $k>2$ diverge and cannot be used to obtain bounds.

${ }^{12}$ R. G. Gordon, J. Math. Phys. 9, 655 (1968). 